\title{
Functional Active Genomics of Isoprene Biogenesis and resistance to cardiovascular diseases
}

Fedoriv Anna

Charity Science Foundation "The New Model of Nature", Head, Chernivtsi, Ukraine. Nanobiotech Research Lab, Biochemist, Chernivtsi, Ukraine annafedoriv5@gmail.com;

Fedoriv Ivan, Nanobiotech Research Lab, Head, Independent Biotechnology Professional, Chernivtsi, Ukraine Charity Science Foundation "The New Model of Nature", Biochemist, Chernivtsi, Ukraine.

ivfedoriv@ukr.net;

\begin{abstract}
The pathologic development of the atherosclerotic process is often associated with the metabolism of saturated and unsaturated fatty acid. Substitution of the saturated fatty acids in nutrition for polyunsaturated fatty acids is traditionally associated with the lowering of risk of coronary breaches rise. Understanding the molecular mechanisms of the atherosclerosis development and progress is very important for early diagnostic and effective medical treatment of the above-mentioned disease. After a thorough analysis of the data available on the pathological atherosclerotic process, we have come to the conclusion that this disease begins from vascular smooth muscle cell (VSMC) impaired function. In the basis of the atherosclerosis development lies isoprenes biogenesis breach, caused by cholesterol and the products of its metabolism. Atherosclerosis is a chronic inflammatory disease of the media wall of large- and medium-sized arteries. And endothelium injury is a consequence of the pathologic process progressing in myocytes. Metabolic problems have become so relevant that it is time to form a metabolic policy. Real target programs for the prevention of the development of metabolic diseases and their diagnostics in the early stages of development should be developed. But in order to achieve this goal, it is necessary to know the real molecular mechanism of development of the early stages of metabolic diseases. It is necessary to recognize that the research work on the metabolic problem was carried out mainly in the plane of the functionally-energy parameter and captures only the consequences of the pathological process. And the very reason and early stages of metabolic diseases remained hidden from us, as they are depending on the pathology in the plane Regulatory, Information, Coordination and Functional active bioenergy system.
\end{abstract}

Keywords: Functional active genomics, isoprene biogenesis, functional active Genomics of Bioethereal Substance, atherosclerosis, resistance, bioenergy system, ether substance. 
Today Atherosclerotic disease is the only major disease that cannot be prevented, stopped or cured. Over the past 50 years, all Atherosclerosis research effort has failed to find a cure. Treatment is mainly symptomatic. Medical interventions are principally targeted at lowering cholesterol levels and reducing platelet activity, as well as treating, for example, hypertension and arrhythmias. For many years the cardiovascular disease has been a major cause of morbidity and mortality across the developed world, and its incidence is also increasing in many developing countries. Indeed, it has recently been shown that even Egyptian mummies, representing the more affluent members of their society several thousand years ago, had established atherosclerosis in multiple blood vessels. ${ }^{1}$ Cardiovascular disease has been extensively researched and has been the subject of considerable media interest and campaigns to promote lifestyle changes, such as stopping smoking, reducing alcohol consumption, avoiding obesity and taking regular exercise. In spite of the many advances in our understanding of the pathogenesis of the atherosclerotic disease, cardiovascular disease remains responsible for nearly half of all deaths in Europe. $^{2}$ The same report highlights the fact that cardiovascular disease is estimated to kill over two million people in the EU and to cost the EU economy some $€ 192$ billion a year. The bulk of these costs relate to healthcare $(57 \%)$, but there are also significant losses of productivity $(21 \%)$ and informal care costs $(22 \%)$. Add to this the UN predictions that the number of people over 65 years of age in the EU is due to increase by some $45 \%$ over the next two decades and it is clear that our health economies face an unprecedented challenge for the future. ${ }^{2,3}$

\section{Objective and methodology}

A major underlying cause of cardiovascular disease is atherosclerosis - a chronic inflammatory disease of the large arteries. Despite substantial advances over the past few decades, our understanding of the molecular mechanisms that link cardiovascular risk factors to the development and progression of atherosclerosis is incomplete. ${ }^{14}$ We divided the development of the pathological atherosclerotic process into 2 stages, and the basis of the division was taken into account the fact that the process itself begins in the smooth muscle cells of blood vessels for the accumulation of oleates, and during the progression of atherosclerosis, the accumulation of cholesterol esters inside the fibrous plaque (mainly linoleates) is noted. ${ }^{4}$ No wonder atherosclerosis is also called a combined disease with changes in the intimidation and media of the artery. The pathologic development of the atherosclerotic process is often associated with the metabolism of saturated and unsaturated fatty acid. Substitution of the saturated fatty acids in nutrition for polyunsaturated fatty acids is traditionally associated with the lowering of risk of coronary breaches rise. Understanding the molecular mechanisms of the atherosclerosis development and progress is very important for early diagnostic and effective medical treatment of the above-mentioned disease. After a thorough analysis of the data available on the pathological atherosclerotic process, we have come to the conclusion that this disease begins from vascular smooth muscle cell (VSMC) impaired function. In the basis of the atherosclerosis development lies isoprenes biogenesis breach, caused by cholesterol and the products of its metabolism. Atherosclerosis is a chronic inflammatory disease of the media wall of largeand medium-sized arteries. And endothelium injury is a consequence of the pathologic process progressing in myocytes. In the basis of the atherosclerosis development lies isoprenes biogenesis breach, caused by cholesterol and the products of its metabolism. ${ }^{5-10}$ 
It is stated that vital processes are not limited only with the existance of ether substance. The systematic and comprehensive approach to the problem of metabolism has ensured the forming of a new model of nature. The living matter is the specific category for the cognition of the objective reality which exists in the form of two substances: ethereal (functional active) and physical one. The existence of the living matter depends on the state of biogenesis of the isoprenoids which display themselves in the specific form of matter or intermediate ethereal medium (bioethereal substance). According to this model the nature is a composite or combined creature.

With the help of the isoprenoids the matter goes over from the physical substance to the ethereal one and this is why it is usual for the nature to have all the real existent and essential properties such as connections and forms of moving. The realization of this machinery contradicts the mechanical model of nature and assists further with the forming of a new model of nature on the base of isoprenoical conception of the development and early stages of metabolic diseases. The notion of the ethereal substance gives the opportunity to deeper discloses the essence of physical and chemical laws, which are absolute in them.

Only continued work on atomic structures and the biophysics of molecular interactions and reactions, will reveal the mechanisms required to understand the essence of physiology / pathology, resistance to metabolic diseases and classical molecular mechanisms by which a metabolic diseases at early stages develop.

\section{Discussion and Findings :}

Metabolic problems have become so relevant that it is time to form a metabolic policy. Real target programs for the prevention of the development of metabolic diseases and their diagnostics in the early stages of development should be developed. But in order to achieve this goal, it is necessary to know the real molecular mechanism of development of the early stages of metabolic diseases.It is necessary to recognize that the research work on the metabolic problem was carried out mainly in the plane of the functionally-energy parameter and captures only the consequences of the pathological process. And the very reason and early stages of metabolic diseases remained hidden from us, as they are depending on the pathology in the plane Regulatory, Information, Coordination and Functional active bioenergy system. 


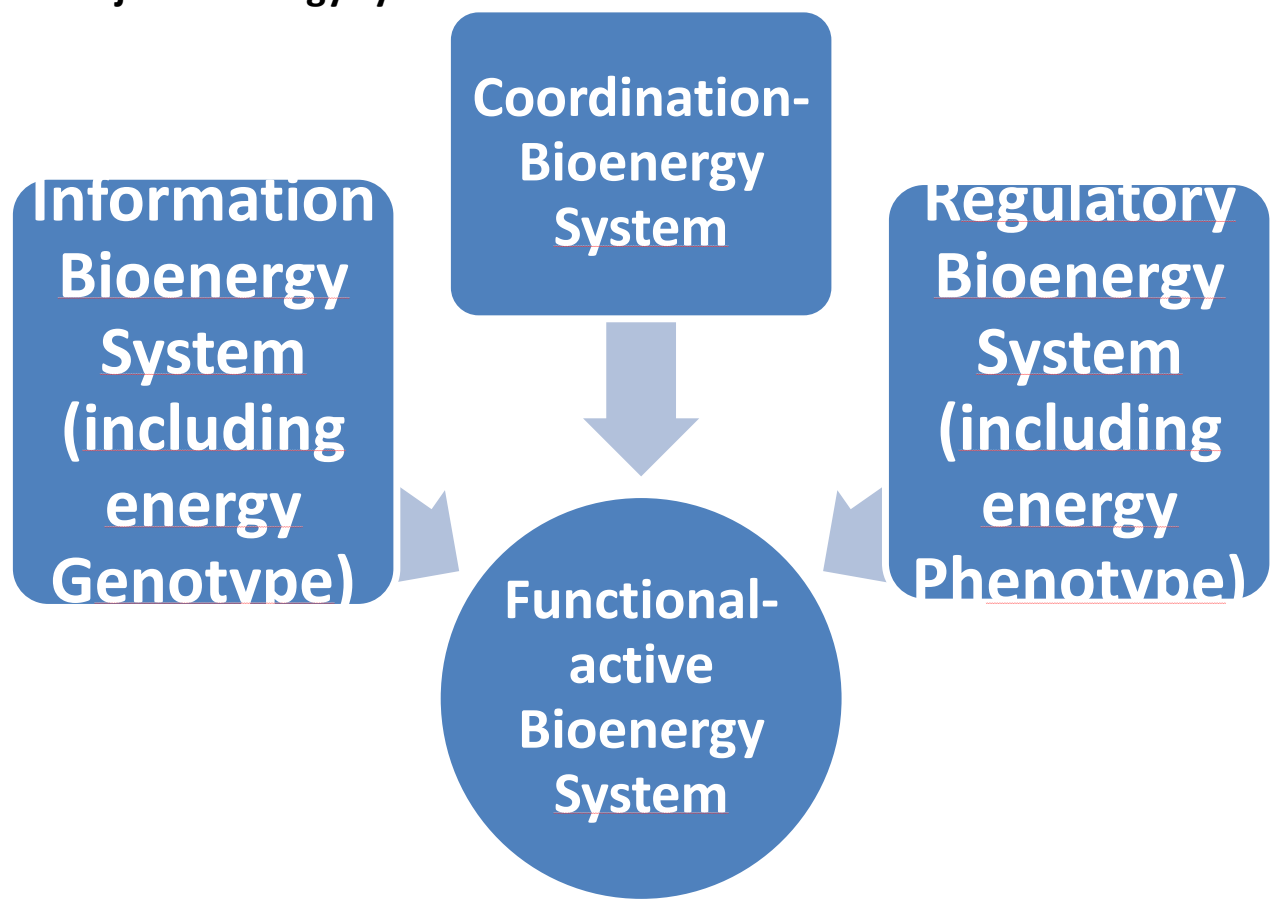

Biogenesis of Isoprenoids, which dominates our work, is part of the process of Functional active and parallel Coordinating bioenergy system and its a stabilizer of organism bioenergetics. ${ }^{8}$ Polyprenyls act as ubiquinone substrates for redox reactions in respiration and photosynthesis. Polyprenyl chains are also present in glycosylphosphatidylinositol lipids used to anchor peripheral membrane proteins.

The stabilising of exocellular proteolysis plays a main role in the development of cellular immunity. Our approach to the regulation of HMG-CoA-reductase has highlighted the functional role of human cholesterol-esterase in the mechanism of isoprene-dependent extracellular proteolysis. Regulation of these reactions could be focal points in influencing this key pathway in the biosynthesis of isoprenes and an attractive pharmacotherapeutic target in the management of tRNA(i)n biosynthesis, functional activity of the cells membranes receptor complexes and biological regulation of extracellular proteolysis and resistance to CVD. A some control points for these processes are the regulatory proteins dolichol kinase and Human cholesterol-esterase > Cholesterol-stearate > Cholesterollinoleate $>$ Cholesterol-palmitate $>$ Cholesterol-stearate $>$ Cholesterol-oleate (HMG-CoAreductase inhibitors). The ethers of oleic acid have demonstrated the lowest rate of hydrolysis. Other than aiding in understanding the natural progression of the extracellular proteolysis, this concept opens the door for a line of novel means of selective immunomodulation protector which may represent new treatment strategies. This may well occur through classical mechanisms by which a metabolic diseases at early stages develop. ${ }^{4}$

At the Nanobiotechnology Research Lab we have put together a unique and comprehensive capability for diagnostics of extracellular proteolysis, early stages of autoimmune processes research, dysfunction in mitochondrial energy metabolism. Some of our researches of diagnostics include:

The functional active Genomics of Bioethereal Substance Test.

The functional active Genomics of Isopentenyl tRNAs Test. Patent \#56396, Ukraine.

Extracellular proteolysis Test ${ }^{\mathrm{TM}}$. 
With the help of these new techniques our company will help our customers compress the time ittakes to bring a drug from discovery through regulatory approval. For engineering and introduction of these methods of diagnostics in the international practice and them commercialization it is necessary to us to using the latest tools, guarantee best prices and standards in quality and service. ${ }^{11-13,15}$ Methods by which specific biomarkers are used for risk detection, diagnosis, prognosis and monitoring of CV diseases. Non-invasive diagnostic test of choice would expand opportunities for testing in hard to reach populations and facilitate diagnosis in physician's offices, satellite clinics, outpatient facilities and other nontraditional testing facilities and would reduce the overall costs of CVD patient care and the healthcare system in general.

\section{Conclusion and recommendations}

Research in the life sciences is becoming multidisciplinary and it is therefore essential that we join forces in order to attain a global vision for the physical and ethereal (functional active substances) basis of the lateral heterogeneity and the heterogeneous organization of cellular membranes in lateral domains of different lipid and protein composition. The existence of different forms and levels of structure organizations in animate and dead nature are akin to such notion as the trigger-property of the matter. Extracellular proteolysis that was formerly considered an "inert" process is gradually perceived as a dynamic disorder in which proteolytic and immune system components play a major role. The stabilizing of extracellular proteolysis plays a main role in the development of cellular immunity

References(the separate publications):

[1] Allam AH, Thompson RC, Wann LS, et al., Computed tomographic assessment of atherosclerosis in ancient Egyptian mummies, JAMA, 2009; 302(19): 2091-4. Crossref | PubMed.

[2] Allender S, Scarborough P, Peto V, et al., European Cardiovascular Disease Statistics, 2008 edition, European Heart Network.

[3] Andrew Carson, European Cardiology 2009;5(2):86-8.4.

[4] Fedoriv A.M., Fedoriv I.V. Atherosclerotic Endothelium Injury is a Consequence of Pathologic Processes in Myocytes // Data of Scientific Research (D.S.R.) 1997. v 6-7.-p.11-14.

[5] Fedoriv A. et al. Functional Genomics of Bioethereal Substance and Resistance to Metabolic Diseases. The first European conference of the ESF Programme in Functional Genomics. Prague,2003.

[6] Fedoriv A. et al. Stabilization of protein prenylation is a novel approach to the prevention and treatment of cancer. International Conference on Tumor Progression and Therapeutic Resistance. November 8-9, 2004, Hilton Philadelphia City Avenue, Philadelphia, PA.

[7] Fedoriv A., Fedoriv I. Membrane Metabolism of Cholesterol. Data of Scientific Research (D.S.R.) 1-2, 58-59, 1998.

[8] Fedoriv A. et al. Information Power Flow and Molecular Mechanism it Correction in Biosystems. Data of Scientific Research (D.S.R.) 3, 88-90, 1999.

[9] Fedoriv A. et al. Isoprene Biogenesis is a Stabilizer of Telomerase Activity and Organism's Bioenergy. Data of Scientific Research (D.S.R.). 2, 95-97, 2000. 
[10] Fedoriv I. et al. Regulatory interrelations between biosynthesis cycles of purines and histidine in cancerogenesis. Data of Scientific Research (D.S.R.). 1, 111-113, 2001.

[11] Fedoriv A. et al. The Isoprenoids Display Themselves in a Specific Form of Matter. / EURESCO Conference. Membrane Dynamics in Endocytosis /Molecular Regulation/, October 6-11,2001, EMBO, Tomar, Portugal.

[12] Fedoriv I. Isoprene Biogenesis Secures Possibility Interaction Between Microfilament System and Cell Membrane. /Microfilament Function and Regulation in Cell Polarity /CNSR/Jacques Monod/EMBO Conference. September 15-19, 2001, Institut Curie, Giens, France.

[13] Fedoriv S. et al. Isoprene Biogenesis, Cancer, AIDS and Revitalize of the Organism. /EURESCO Conference. Cellular and Molecular Basis of Regeneration. 31 August - 5 September 2002. Castelvecchio Pascoli, Italy.

[14] Huang A, Patel S, McAlpine CS, Werstuck GH. Huang A, et al. The Role of Endoplasmic Reticulum Stress-Glycogen Synthase Kinase-3 Signaling in Atherogenesis. Int J Mol Sci. 2018 May 30;19(6):1607. doi: 10.3390/ijms19061607. Int J Mol Sci. 2018. PMID: 29848965 Free PMC article. Review.

[15] Fedoriv A. et al. Functional Genomics of Bioethereal Substance and Resistance to Metabolic Diseases. The first European conference of the ESF Programme in Functional Genomics. Prague,2003. 